\title{
Investigating Functional Dyspepsia in Asia
}

\author{
Yeong Yeh Lee ${ }^{1}$ and Andrew Seng Boon Chua ${ }^{2 *}$ \\ ${ }^{1}$ Department of Medicine, School of Medical Sciences, University Sains Malaysia, Kubang Kerian, Kelantan, Malaysia; and ${ }^{2}$ Gastro Centre Ipoh, \\ Ipoh, Perak, Malaysia
}

\begin{abstract}
The diagnosis of functional dyspepsia (FD) is challenging since it depends largely on symptoms which are often heterogeneous and overlapping. This is particularly so in Asia with many different cultures and languages. Symptom-based diagnosis of FD based on Rome III criteria has not been fully validated and it may not be suitable in some Asian populations. Clinicians often assume that investigations in FD are not rewarding and physiological tests are often not available unless in the research setting. Investigation of alarm features and role of Helicobacter pylori in FD remain controversial but experts agreed that both should be tested. Physiological tests including gastric accommodation and chemical hypersensitivity tests are underutilized in Asia and available studies were few. While experts do not recommend routine clinical use of gastric accommodation tests but they agree that these tests can be advocated if clinically indicated. Empiric therapeutic trial is not currently a diagnostic option. The pathogenesis of FD is still poorly understood and there is a substantial placebo response. As a conclusion, a diagnosis of FD is challenging especially so in the context of Asia and despite the limitations of available physiological tests experts agreed that these tests can be advocated if and when clinically indicated.
\end{abstract}

(J Neurogastroenterol Motil 2012;18:239-245)

Key Words

Asia; Functional dyspepsia; Helicobacter pylori

\section{Introduction}

To define dyspepsia based on a constellation of symptoms has been controversial particularly so in Asia due to cultural heterogeneity across different populations. This poses a challenge to the diagnosis of functional dyspepsia (FD) since at the moment the diagnosis largely depends on symptomatology and exclusion of organic gastrointestinal diseases. ${ }^{1}$ Furthermore there is considerable overlap in symptoms between FD, non-erosive reflux disease (NERD), irritable bowel syndrome (IBS) and Helicobacter pylori dyspepsia.
Clinicians often consider investigations in FD as non-rewarding due to low yields and physiological tests are not always available other than in a research setting. This was recently revealed in a survey conducted among 43 physicians and researchers on their current practice of functional gastrointestinal disorders during the first Asian Pacific Topic Conference at Tokyo in November $2010 .^{2}$ This was followed by a more recent publication on the consensus statement of FD in Asia. ${ }^{3}$

The current paper reviewed on some of the controversies surrounding investigations of FD with focus in Asia. Among others, this review discussed on the weaknesses of symptom-based diagnosis of FD, investigations of alarm features, role of $H$. pylori and

Received: March 26, 2012 Revised: May 1, 2012 Accepted: May 8, 2012

(c) This is an Open Access article distributed under the terms of the Creative Commons Attribution Non-Commercial License (http://creativecommons. org/licenses/by-nc/3.0) which permits unrestricted non-commercial use, distribution, and reproduction in any medium, provided the original work is properly cited.

*Correspondence: Andrew Seng Boon Chua, MD

Gastro Centre Ipoh, 31 Lebuh Taman Ipoh, Ipoh Garden South, 31400 Ipoh, Perak, Malaysia

Financial support: None.

Tel: +60-05-545-8488, Fax: +60-05-545-7488, E-mail: drandrewchua@gmail.com

Conflicts of interest: None. 
the practicality of physiological tests with reference to recent publications on current practice survey and consensus statement.

\section{Symptom-based Diagnosis of Functional Dys- pepsia}

Dyspepsia or commonly known as indigestion did not have an agreed definition until in the late 1980s. ${ }^{4}$ With Rome I in 1991, functional (non-ulcer) dyspepsia was defined as chronic dyspepsia (epigastric or retrosternal symptoms present in at least $25 \%$ of the time for at least 4 weeks) in the absence of investigated organic disease. While this definition is now widely accepted it is also recognised that symptoms may be perceived differently within different cultures and countries. This was further refined in Rome II which defined FD as the presence of pain or discomfort centred in the epigastrium and present for at least 12 weeks over the last 12 months and not explained by upper gastrointestinal investigation. ${ }^{5}$ Within Asia, the Rome II criteria for diagnosis of FD have been validated and this was shown in a factor analysis of symptoms involving 1,012 subjects across nine Asian regions. ${ }^{6}$

Some clinicians consider the time frame imposed in the Rome II criteria as restrictive. In addition, a factor analysis on symptoms suggests that there is a meal-related syndrome not accounted for by Rome II. Under the revised Rome III criteria in 2006, a diagnosis of FD requires symptoms to be present for the last 3 months with symptom onset at least 6 months before diagnosis. ${ }^{7}$ It also proposed two distinct subgroups under FD which comprise the postprandial distress syndrome and epigastric pain syndrome. There is on-going effort to translate and validate the Rome III Diagnostic Questionnaires into different languages within Asia. ${ }^{8}$ While a study from Korea supports the use of Rome III criteria in FD, another recent study from Japan suggests that 6-month period after symptom onset could miss the diagnosis in their population. ${ }^{9,10}$ In a community study from Korea, the proportion of postprandial distress syndrome was $47 \%$, epigastric pain syndrome was $26 \%$ and $27 \%$ was overlap syndrome. ${ }^{9}$

With the shift on definitions of FD from Rome I to the current Rome III, one message is clear. Symptoms are poor predictor of FD and significant overlaps are often seen with IBS and NERD. In a study from China, the overlap between FD and IBS was observed in $5 \%$ of gastroenterology clinic patients with an odd ratio of 2.09 and they often had higher severity scores for postprandial fullness symptom. ${ }^{11}$

The Asian consensus on FD agreed to include bloating as one of the symptoms since clinically it is one of the more com- monly reported symptom. Only $5 \%$ of members in the consensus agreed with a 6-month period of symptoms in Rome III but most members agreed that 3 months were enough. ${ }^{3}$ For research purpose, the period of 6-month in Rome III is followed. More cross-cultural studies are needed within the Asian region using the Rome III criteria.

\section{Endoscopy and Investigation of Alarm Fea- tures}

Uninvestigated dyspepsia (UD) refers to patients with dyspeptic symptoms but did not undergo investigations to rule out peptic ulcer and gastric cancer. Generally accepted alarm features include age above 50 years, family history of upper gastrointestinal cancer, unintended weight loss, gastrointestinal bleeding, progressive dysphagia, odynophagia, unexplained iron deficiency anaemia, persistent vomiting, palpable mass or lymphadenopathy and jaundice. Alarm features are useful in identifying high risk subjects who need early endoscopy. While data from Asia are scanty but available studies suggest that the frequency of UD varies between $8 \%-30 \% .^{12}$ Most Asian patients with UD commonly have FD with organic causes detected in approximately $30 \%-40 \%$. Older age appears to be an important predictor for the presence of organic diseases particularly in those above 50 years old. In 1985 the American College of Physicians published a guideline which prompted a referral for endoscopy in those patients with dyspepsia over the age $45 .{ }^{13}$ This age threshold was later revised to 50 years old by the World Congresses of Gastroenterology which matched the screening age for colon cancer. ${ }^{14}$ While the overall yield from endoscopy is low but there are a number of benefits in performing endoscopy. These benefits include an exclusion of gastric cancer, increase of patients' satisfaction and reassurance and decrease in primary care consultations with normal endoscopic findings.

Subjects with organic pathologies may also have functional dyspepsia since their symptoms often overlap with each other. In a large scale Future study involving 9,125 Japanese patients with chronic gastritis surveyed for FD using the Rome III criteria, it was found that clinical characteristics between the two conditions were very similar. ${ }^{15}$ In addition, patients with $\mathrm{FD}$ often have persistent symptoms and may return for a repeat upper endoscopy. A study suggests that $9 \%$ of patients who underwent repeat upper endoscopy for dyspepsia usually do so at a median of 1.7 years from the initial endoscopy. ${ }^{16}$ The yield was modest and lower than the yield at initial endoscopy. Even though several studies have suggested that alarm features may have a lower predictive 
value for diagnosis of organic pathologies but most physicians still advocate further investigations. ${ }^{3}$

If clinically indicated as suggested in the recent Asian consensus on FD, complete blood count and biochemistry including creatinine, electrolytes, sugar, thyroid and liver function may be useful. ${ }^{3}$ In Asian countries with high parasitic burden, stool examination for parasites and faecal blood test are indicated. Ultrasound and CT scan of abdomen can be employed in subjects with suspected liver cancers and to rule out biliary pathology.

\section{Role of Helicobacter pylori Infection}

Compelling evidence exists for the role of $H$. pylori in the causation of peptic ulcer disease and gastric cancer, both implicated with dyspepsia. ${ }^{17}$ Most global burden of $H$. pylori infection comes from Asia and therefore exclusion of this bacterium is an important part of diagnostic exercise in any Asian patients presented with dyspepsia to their physicians. The eradication of H. pylori is effective in alleviating symptoms in ulcer disease but the evidence for eradication in non-ulcer dyspepsia has been less straightforward. ${ }^{18}$ Eradication in non-ulcer dyspepsia appears to improve dyspeptic symptoms more in the Chinese population than in Western populations. ${ }^{19}$ Eradication rates were less likely to be successful in non-ulcer dyspepsia and this is presumably due to a less dense bacterial colonisation in non-ulcer dyspepsia and that a less severe gastritis does not allow penetration of antibiotics. $^{18}$

There is increasing evidence that the gut-brain-microbial axis plays an important role in functional gut disorders with most reported studies coming from IBS. ${ }^{20,21}$ Post- infectious FD has been described following gastrointestinal infection and is characterized by persistent mucosal inflammation in the duodenum. ${ }^{22}$ While epidemiological studies suggest a link between $H$. pylori infection and functional dyspepsia but the mechanisms of this link remain to be elucidated. ${ }^{23}$ Some biological biomarkers have shown promise in showing this pathophysiological link including an abnormal levels of ghrelin or leptin and the altered expression of muscle-specific microRNAs. ${ }^{24-26}$ The site of pathology may actually lies in the duodenum and not in the stomach as one would have believed. Gastric motility and hypersensitivity testing in $\mathrm{H}$. pylori-dyspepsia showed conflicting results. ${ }^{27}$ On the other hand, abnormal motility responses and inflammatory cells including eosinophils and intra-epithelial lymphocytes were more commonly found in the duodenum in $H$. pylori-dyspepsia.

In the survey, only $58 \%$ of physicians in Asia had checked for the status of $H$. pylori infection with almost a similar proportion of physicians advocating eradication in positive cases. ${ }^{2}$ The consensus members agreed that $H$. pylori status should be checked. ${ }^{3}$ However the evidence was at the most moderate for the role of $H$. pylori in the pathogenesis of FD. It was proposed by some consensus members that $H$. pylori-dyspepsia should be a separate entity from FD since gastritis can now be identified easily with advanced endoscopic techniques and that $H$. pylori-dyspepsia is a form of post-infectious FD.

\section{Gastric Accommodation Testing}

While heterogeneous, the delay in gastric emptying and heightened hypersensitivity to gastric distension are important mechanisms underlying $\mathrm{FD}^{28}$ Gastric accommodation is a vagally mediated reflex allowing a reduction in the proximal gastric tone but an increase in the compliance and not pressure in response to food intake. ${ }^{29}$ This reflex was shown to be impaired in FD resulting in early satiety and weight loss.

Gastric barostat is the earliest technique used for investigating gastric accomodation and is the current gold standard. Studies using gastric barostat had shown that accommodation response was impaired in $40 \%$ of patients with FD. ${ }^{30}$ This method is however invasive, time consuming and not comfortable to patients. Other newer techniques include single photon emission computed tomography (SPECT), ${ }^{31}$ 2- and 3-dimensional gastric ultrasound $^{32}$ and magnetic resonance imaging. ${ }^{33}$ These imaging techniques which are often sophisticated can allow researchers to quantitate the fasting and postprandial gastric volumes in a reliable and reproducible fashion. While non-invasiveness is an advantage but the cost, sophistication and radiation currently limit the use of these techniques in clinical practice. Drinking test is a simpler test to perform and more applicable in a routine clinical setting. ${ }^{34}$ The principle of the test is based on the assumption that gastric volume is reduced with impaired gastric accommodation and therefore limits the drinking volume. This test has been validated against the gastric barostat but the reproducibility is limited due to differences in types of drink and rates of drinking.

In a survey conducted among physicians in Asia, only $37 \%$ of patients with suspected FD were examined for upper gastrointestinal motility. Gastric barostat and drinking test were used in only $16 \%$ of patients. ${ }^{2}$ The Asian consensus statement on FD states that gastric sensorimotor function tests including the gastric accommodation test may be useful in some subgroups of patients such as diabetic gastroparesis and generalized gastrointestinal disorders but are not currently recommended as routine clinical tests. ${ }^{3}$ 


\section{Chemical Hypersensitivity Test}

Heightened visceral hypersensitivity in response to a meal or nutrient stimuli is a key pathophysiological link to dysmotility symptoms in FD in particular belching, early satiety and weight loss. It has been shown that duodenal infusion of lipid in subjects with FD increased gastric distension and symptoms in a dose dependent fashion. ${ }^{35}$ The relief of symptoms following administration of loxiglumide, a cholecystokinin (CCK) A receptor antagonist suggests that $\mathrm{CCK}$ release following a lipid stimuli is the mediator of gastric hypersensitivity in FD. ${ }^{36}$ And since FD is a diagnosis of exclusion, using CCK infusion as a challenge test is appealing. It has been shown that infusion of CCK octapeptide (CCK-8) at $6 \mathrm{ng} / \mathrm{kg}$ per minute reproduces their dyspeptic symptoms in the majority of patients with FD. ${ }^{37}$ Similar infusion of the CCK-8 in healthy controls and peptic ulcer patients do not produce any significant complaints. The test is safe and reproducible but experience from Asia is extremely limited partly because of the invasive nature of the test.

Another useful test is the Buspirone challenge test. ${ }^{38}$ Buspirone is a serotonin (5-hydroxytryptamine, 5-HT) $1 \mathrm{~A}$ agonist and acts at the hypothalamic level to stimulate the release of prolactin. The extent of prolactin release from the Buspirone challenge is a reliable measure of central 5-HT sensitivity which can be impaired in patients with FD. 5-HT is an important neurotransmitter which modulates many gastrointestinal physiological tasks including pain perception, control of eating and sensorimotor function. It has been reported that greater prolactin responses (indicating hypersensitive central serotoninergic receptors) were found in patients with FD than in healthy controls or patients with peptic ulcer disease with Buspirone challenge. ${ }^{39,40}$ Again experience with this test in Asia has been extremely lacking and therefore it has not been mentioned in the survey or the consensus statement.

A more recent report suggested that oral capsaicin load of $0.75 \mathrm{mg}$ induced dyspeptic symptoms in half of subjects with FD but negligible in placebo. ${ }^{41}$ Capsaicin, a well-known compound present in chili is a potent activator of transient receptor potential ion channel of the vanilloid type 1 which has been recognized to play an important role in visceral nociception. Whether this test is suitable for the Asian population remains to be investigated. It is known that chili consumption in Asian population is much higher than the western population. The prevalence of gastroesophageal reflux symptoms appears lower in populations from Thailand, China and Iran whose diets have included chili and spicy food but not in the Korean population ${ }^{42}$ but no data is available for the role of chili diet in FD.

As it stands, the Asian consensus on FD acknowledges that visceral hypersensitivity to nutrient stimuli may play a role in the pathogenesis of FD but the grade of evidence is considered low. ${ }^{3}$ There is no mention of any chemical challenge tests in the consensus. None of these tests were used among physicians in Asia when surveyed for their current practice in the management of FD. ${ }^{2}$

\section{Pharmacologic Trial as a Means of Diagno- sing Functional Dyspepsia}

Empiric therapeutic trial can be a reliable means of diagnosis when faced with diagnostic uncertainty in diseases including gastro-oesophageal reflux disease. While this approach can be successful in gastroesophageal reflux disease but this is not the case with FD. There are a few reasons for this.

Firstly, the pathogenesis of FD remains, for the most part not fully established or understood. Secondly, few if any treatments currently available is really effective for FD. Use of proton pump inhibitor can provide symptoms relief in FD among the Western populations but the few available studies from Asia are not convincing. ${ }^{43}$ Prokinetics including domperidone, itopride, mosapride, levosulpride and cinitapride have been tried in clinical studies and appear clinically efficacious. However the studies reported were often heterogenous in methodologies and small in size. $^{44}$ The new drug, acotiamide, an acetylcholinesterase inhibitor is promising and has been shown to be efficacious and safe in the elimination of meal-related FD symptoms. ${ }^{45}$ Thirdly, there is often a substantial placebo response in any treatments. The mechanism of placebo effect is unclear but a study had shown that clinical improvement in functional disorders was independent of changes in the gastroduodenal motility or gastric hypersensitivity to distension. ${ }^{46}$

With all of the above problems with pharmacological interventions in FD, it is of no surprise that in the Asian FD consensus an integrated approach to management is highly recommended instead of pharmacological therapy alone. ${ }^{3}$ The physiological, biological, psychological and social factors should be explored in all patients with FD. In the survey of current practice in FD, almost all of physicians (90\%) would give advice on lifestyle changes and only half would prescribe medications at first visit. ${ }^{2}$ Most of physicians (89\%) would prescribe proton pump inhibitor and all physicians would prescribe prokinetics at some point. 


\section{Conclusion}

A list of suggested investigations in the work-up of FD is shown in Table 1 . The list is not exhaustive especially with investigations of alarm features where other blood tests including amy-

Table 1. Investigations in Functional Dyspepsia

\begin{tabular}{ll}
\hline Exclusion of organic diseases & Gastric accommodation test \\
Upper gastrointestinal & Gastric barostat \\
endoscopy & SPECT \\
Ultrasound or CT scan & Gastric ultrasound 2- or \\
abdomen & 3-dimensional \\
H. pylori testing & MRI \\
Alarm features & Drinking test \\
Complete blood counts & Chemical hypersensitivity test \\
Electrolytes, sugar and & Cholecystokinin octapeptide \\
creatinine & infusion test \\
Thyroid and liver function & Buspirone challenge test \\
Faecal blood testing and stool for & Oral capsaicin load test \\
parasites & \\
Colonoscopy & \\
Ultrasound or CT scan & \\
abdomen & \\
\hline H. pylori, Helicobacter pylori; SPECT, single photon emission computed \\
tomography.
\end{tabular}

lase, coeliac serology, C-reactive proteins or tumour markers maybe useful depending on the local prevalence of organic diseases. In the possible near future, biomarkers and genetic markers could prove to be important complementary diagnostic tools in FD. A summary of the recent published Asian consensus statement on $\mathrm{FD}$ in the context of diagnosis and investigations is shown in Table 2.

Most experts in Asia agreed that symptoms and upper gastrointestinal endoscopy are enough to make a diagnosis of FD. ${ }^{3}$ How useful is the Rome questionnaire in diagnosing FD in the Asian context remains to be validated. Also if there is a need for further tests then which tests and can combining tests increase the diagnostic accuracy? Or is there a need to use any diagnostic tests at all since $\mathrm{FD}$ is not a dangerous condition and time itself is a diagnostic tool? All of these questions remain unanswered and would be grounds for future studies. However, most experts agree that alarm features should be investigated and gastric motility tests are advocated if clinically indicated. ${ }^{3}$ While chemical hypersensitivity test has not been mentioned in the consensus but a simple non-invasive test such as oral capsaicin load test may prove useful once the test is validated in the Asian population. Finally, while therapeutic trial is not currently providing a diagnostic option but with better understanding on the pathogenesis of FD and with new promising drugs in the pipeline, therapeutic

Table 2. A Summary of Asian Consensus on Functional Dyspepsia in the Context of Diagnosis and Investigations

\begin{tabular}{|c|c|c|c|c|c|c|c|c|}
\hline \multirow{2}{*}{ No. } & \multirow{2}{*}{ Statement ${ }^{\mathrm{a}}$} & \multirow{2}{*}{$\begin{array}{l}\text { Grade of } \\
\text { evidence }^{b}\end{array}$} & \multicolumn{6}{|c|}{ Level of agreement ${ }^{\mathrm{c}}(\%)$} \\
\hline & & & a & $b$ & c & d & e & $f$ \\
\hline 4 & $\begin{array}{l}\text { A diagnosis of FD can be considered on the basis of clinical } \\
\text { symptoms and upper gastrointestinal endoscopy results. }\end{array}$ & Moderate & 78.9 & 15.8 & 5.3 & 0.0 & 0.0 & 0.0 \\
\hline 5 & $\begin{array}{l}\text { Dyspepsia patients with alarm features should be investigated } \\
\text { before the diagnosis of FD is accepted. }\end{array}$ & High & 94.7 & 5.3 & 0.0 & 0.0 & 0.0 & 0.0 \\
\hline 6 & $\begin{array}{l}\text { Other useful investigations for dyspepsia include complete blood } \\
\text { count and blood biochemistry tests. Patients with dyspepsia } \\
\text { should be tested for H. pylori. Stool examination for parasites in } \\
\text { areas with high prevalence of infestations and fecal blood testing } \\
\text { are also useful. Upper abdominal ultrasound or CT scan may be } \\
\text { employed if indicated clinically. }\end{array}$ & Moderate & 52.6 & 36.8 & 10.5 & 0.0 & 0.0 & 0.0 \\
\hline 7 & $\begin{array}{l}\text { Gastric sensorimotor function tests including gastric emptying or } \\
\text { accommodation studies may be useful in some subgroups of } \\
\text { patients but are not recommended as routine clinical tests. }\end{array}$ & High & 84.2 & 10.5 & 5.3 & 0.0 & 0.0 & 0.0 \\
\hline
\end{tabular}

${ }^{a}$ Twenty-two consensus members from Asian countries were recruited based on their scientific merits on FD. They were divided into 4 teams based on definitions and diagnosis, epidemiology, pathophysiology and management. Each team would generate consensus statements based on current literature reviews. ${ }^{\mathrm{b}}$ Grade of evidence based on GRADE Working Group is as follows: high, further research is unlikely to change our confidence in the estimate of effect; moderate, further research is likely to have an important impact on our confidence in the estimate of effect and might change the estimate; low, further research is very likely to have an important impact on our confidence in the estimate of effect and is likely to change the estimate; and very low, any estimate of effect is very uncertain. ${ }^{c}$ Level of agreement is as follows: a, accept completely; b, accept with minor reservation; c, accept with major reservation; d, reject with major reservation; e, reject with minor reservation; and f, reject completely. FD, functional dyspepsia; H. pylori, Helicobacter pylori. 
trial can be a diagnostic option after all.

As a conclusion, diagnosis of FD is challenging due to the heterogenous and overlapping nature of symptoms especially in Asia with many different cultures and languages. Despite the limitations of certain available investigative tools in $\mathrm{FD}$ most experts in Asia agreed that such investigations should be carried out if and when clinically indicated.

\section{References}

1. Chua AS. Reassessment of functional dyspepsia: a topic review. World J Gastroenterol 2006;12:2656-2659.

2. Miura S, Sugano K, Kinoshita Y, et al. Diagnosis and treatment of functional gastrointestinal disorders in the Asia-Pacific region: a survey of current practices. J Gastroenterol Hepatol 2011;26(suppl 3):2-11.

3. Miwa H, Ghoshal UC, Gonlachanvit S, et al. Asian consensus report on functional dyspepsia. J Neurogastroenterol Motil 2012;18:150168.

4. Agreus L. Natural history of dyspepsia. Gut 2002;50(suppl 4):iv2iv9.

5. Talley NJ, Stanghellini V, Heading RC, Koch KL, Malagelada JR, Tytgat GN. Functional gastroduodenal disorders. Gut 1999;45(suppl 2):II37-II42.

6. Kwan AC, Bao TN, Chakkaphak S, et al. Validation of Rome II criteria for functional gastrointestinal disorders by factor analysis of symptoms in Asian patient sample. J Gastroenterol Hepatol 2003;18: 796-802.

7. Tack J, Talley NJ, Camilleri M, et al. Functional gastroduodenal disorders. Gastroenterology 2006;130:1466-1479.

8. Lee YY, Waid A, Tan HJ, Chua AS, Whitehead WE. The validity and reliability of the Malay-language translation of the Rome III Diagnostic Questionnaire for irritable bowel syndrome. J Gastroenterol Hepatol 2012;27:746-750.

9. Park H. Functional gastrointestinal disorders and overlap syndrome in Korea. J Gastroenterol Hepatol 2011;26(suppl 3):12-14.

10. Manabe N, Haruma K, Hata J, et al. Clinical characteristics of Japanese dyspeptic patients: is the Rome III classification applicable? Scand J Gastroenterol 2010;45:567-572.

11. Wang A, Liao X, Xiong L, et al. The clinical overlap between functional dyspepsia and irritable bowel syndrome based on Rome III criteria. BMC Gastroenterol 2008;8:43.

12. Ghoshal UC, Sing R, Chang FY, et al. Epidemiology of uninvestigated and functional dyspepsia in Asia: facts and fiction. J Neurogastroenterol Motil 2011;17:235-244.

13. Talley NJ, Vakil NB, Moayyedi P. American gastroenterological association technical review on the evaluation of dyspepsia. Gastroenterology 2005;129:1756-1780.

14. Talley NJ, Axon A, Bytzer P, Holtmann G, Lam SK, Van Zanten S. Management of uninvestigated and functional dyspepsia: a Working Party Report for the World Congresses of Gastroenterology 1998. Aliment Pharmacol Ther 1999;13:1135-1148.

15. Kinoshita Y, Chiba T; FUTURE Study Group. Characteristics of
Japanese patients with chronic gastritis and comparison with functional dyspepsia defined by Rome III criteria: based on the large-scale survey, FUTURE study. Intern Med 2011;50:2269-2276.

16. Ladabaum U, Dinh V. Rate and yield of repeat upper endoscopy in patients with dyspepsia. World J Gastroenterol 2010;16:2520-2525.

17. Goh KL, Chan WK, Shiota S, Yamaoka Y. Epidemiology of Helicobacter pylori infection and public health implications. Helicobacter 2011;16(suppl 1):1-9.

18. O'Morain C. Role of Helicobacter pylori in functional dyspepsia. World J Gastroenterol 2006;12:2677-2680.

19. Jin X, Li YM. Systematic review and meta-analysis from Chinese literature: the association between Helicobacter pylori eradication and improvement of functional dyspepsia. Helicobacter 2007;12:541-546.

20. Lee YY, Chua AS. Influence of gut microbes on the brain-gut axis (Gut 2011;60:307-317). J Neurogastroenterol Motil 2011;17:427429 .

21. Cryan JF, O'Mahony SM. The microbiome-gut-brain axis: from bowel to behaviour. Neurogastroenterol Motil 2011;23:187-192.

22. Li X, Chen $\mathrm{H}, \mathrm{Lu} \mathrm{H}$, et al. The study on the role of inflammatory cells and mediators in post-infectious functional dyspepsia. Scand J Gastroenterol 2010;45:573-581.

23. Bazzoli F, De Luca L, Pozzato P, et al. Helicobacter pylori and functional dyspepsia: a review of previous studies and commentary on new data. Gut 2002;50(suppl 4): iv33-iv35.

24. Osawa H, Nakazato M, Date Y, et al. Impaired production of gastric ghrelin in chronic gastritis associated with Helicobacter pylori. J Clin Endocrinol Metab 2005;90:10-16.

25. Nishizawa T, Suzuki H, Nomoto Y, et al. Enhanced plasma ghrelin levels in patients with functional dyspepsia. Aliment Pharmacol Ther 2006;24(suppl 4):104-110.

26. Saito $Y$, Suzuki $H$, Tsugawa $H$, et al. Dysfunctional gastric emptying with down-regulation of muscle-specific microRNAs in Helicobacter pylori-infected mice. Gastroenterology 2011;140:189-198.

27. Suzuki H, Matsuzaki J, Hibi T. What is the difference between Helicobacter pylori-associated dyspepsia and functional dyspepsia? J Neurogastroenterol Motil 2011;17:124-130.

28. Tack J, Lee KJ. Pathophysiology and treatment of functional dyspepsia. J Clin Gastroenterol 2005;39(5 Suppl 3):S211-S216.

29. Azpiroz F, Malagelada JR. Physiological variations in canine gastric tone measured by an electronic barostat. Am J Physiol 1985;248(2 Pt 1):G229-G237.

30. Azpiroz F, Malagelada JR. Isobaric intestinal distension in humans: sensorial relay and reflex gastric distension. Am J Physiol 1990;258(2 Pt 1):G202-G207.

31. Van den Elzen BD, Bennink RJ, Wieringa RE, Tytgat GN, Boeckxstaens GE. Fundic accommodation assessed by SPECT scanning: comparison with the gastric barostat. Gut 2003;52:1548-1554.

32. Mundt MW, Hausken T, Smout AJ, Samsom M. Relationships between gastric accommodation and gastrointestinal sensations in healthy volunteers. A study using the barostat technique and two- and three-dimensional ultrasonography. Dig Dis Sci 2005;50:1654-1660.

33. Marciani L, Gowland PA, Spiller C, et al. Effect of meal viscosity and nutrients on satiety, intragastrci dilution, and emptying assessed by MRI. Am J Physiol Gastrointest Liver Physiol 2001;280:G1227G1233.

34. Tack J, Caenepeel P, Piessevaux H, Cuomo R, Janssens J. Assess- 
ment of meal induced gastric accommodation by a satiety drinking test in health and in severe functional dyspepsia. Gut 2003;52:12711277.

35. Feinle C, Meier O, Otto B, D'Amato M, Fried M. Role of duodenal lipid and cholecystokinin A receptors in the pathophysiology of functional dyspepsia. Gut 2001;48:347-355.

36. Chua AS, Keeling PW. Cholecystokinin hyperresponsiveness in functional dyspepsia. World J Gastroenterol 2006;12:2688-2693.

37. Chua AS, Dinan TG, Rovati LC, Keeling PW. Cholecystokinin hyperresponsiveness in dysmotility-type nonulcer dyspepsia. Ann N Y Acad Sci 1994;713:298-299.

38. Chua AS, Keeling PW, Dinan TG. Role of cholecystokinin and central serotonergic receptors in functional dyspepsia. World J Gastroenterol 2006;12:1329-1335.

39. Chua A, Keating J, Hamilton D, Keeling PW, Dinan TG. Central serotonin receptors and delayed gastric emptying in non-ulcer dyspepsia. BMJ 1992;305:280-282.

40. Dinan TG, Mahmud N, Rathore O, et al. A double-blind placebo-controlled study of buspirone-stimulated prolactin release in non-ulcer dyspepsia-are central serotoninergic responses enhanced? Aliment
Pharmacol Ther 2001;15:1613-1618.

41. Fuhrer M, Vogelsang H, Hammer J. A placebo-controlled trial of an oral capsaicin load in patients with functional dyspepsia. Neurogastroenterol Motil 2011;23:918-e397.

42. Gonlachanvit S. Are rice and spicy diet good for functional gastrointestinal disorders? J Neurogastroenterol Motil 2010;16:131-138.

43. Wang WH, Huang JQ, Zheng GF, et al. Effects of proton-pump inhibitors on functional dyspepsia: a meta-analysis of randomized placebo-controlled trials. Clin Gastroenterol Hepatol 2007;5:178185.

44. Moayyedi P, Soo S, Deeks J, Delaney B, Innes M, Forman D. Pharmacological interventions for non-ulcer dyspepsia. Cochrane Database Syst Rev 2006;18:CD001960.

45. Matsueda K, Hongo M, Tack J, Saito Y, Kato H. A placebo-controlled trial of acotiamide for meal-related symptoms of functional dyspepsia. Gut 2012;61:821-828.

46. Mearin F, Balboa A, Zárate N, Cucala M, Malagelada JR. Placebo in functional dyspepsia: symptomatic, gastrointestinal motor, and gastric sensorial responses. Am J Gastroenterol 1999;94:116-125. 